\title{
Synthesis of the $\mathrm{FeOOH}$ microtubes with inner
}

\section{surface modified by Ag nanoparticles}

\section{Supporting Information}

Vera V. Strykanova, Larisa B. Gulina,* Valeri P. Tolstoy, Elena V. Tolstobrov,

Denis V. Danilov, Irina Skvortsova

Saint Petersburg State University, 199034, 7/9 Universitetskaya nab., St. Petersburg, Russia

*Corresponding author e-mail: l.gulina@spbu.ru

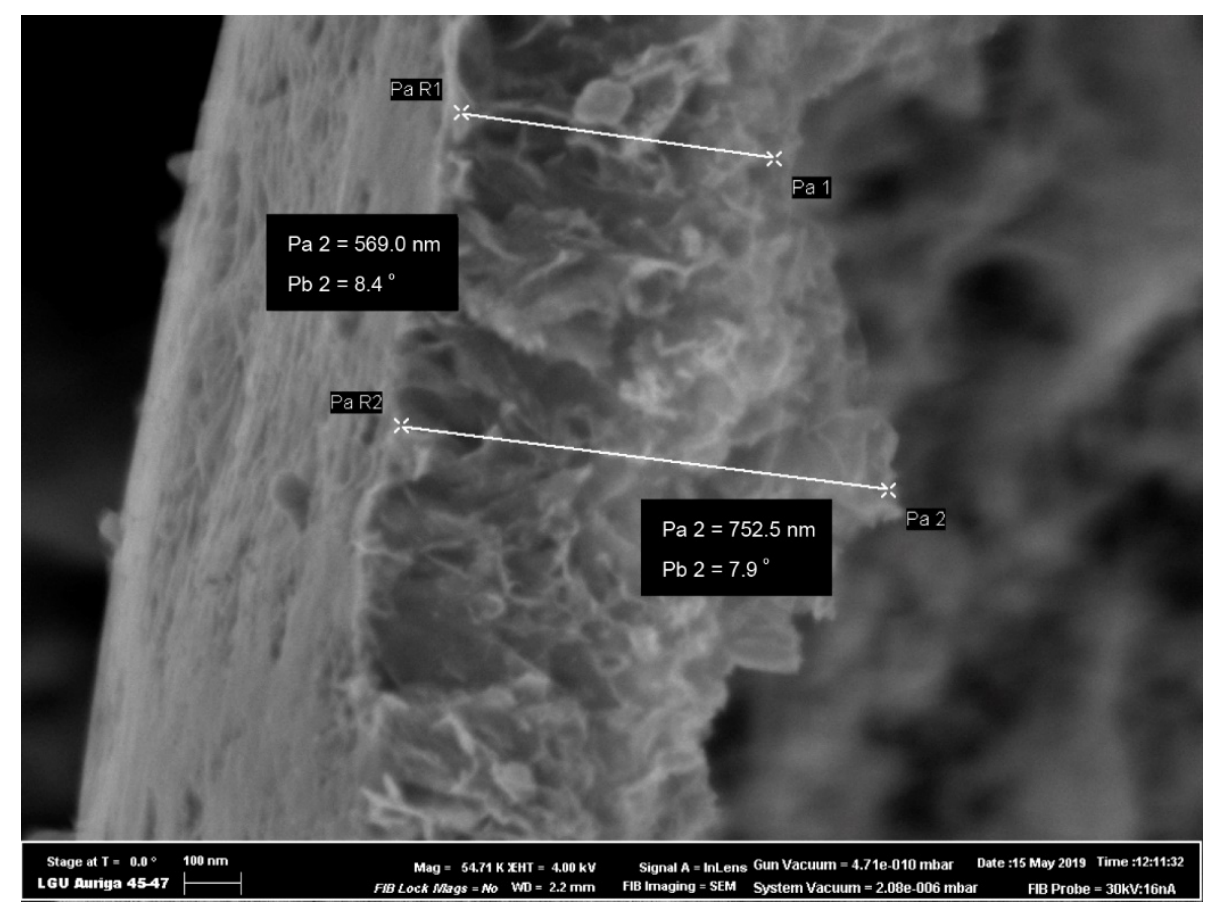

Figure S1. SEM images of interfacial $\gamma$-FeOOH film from cross-view. 


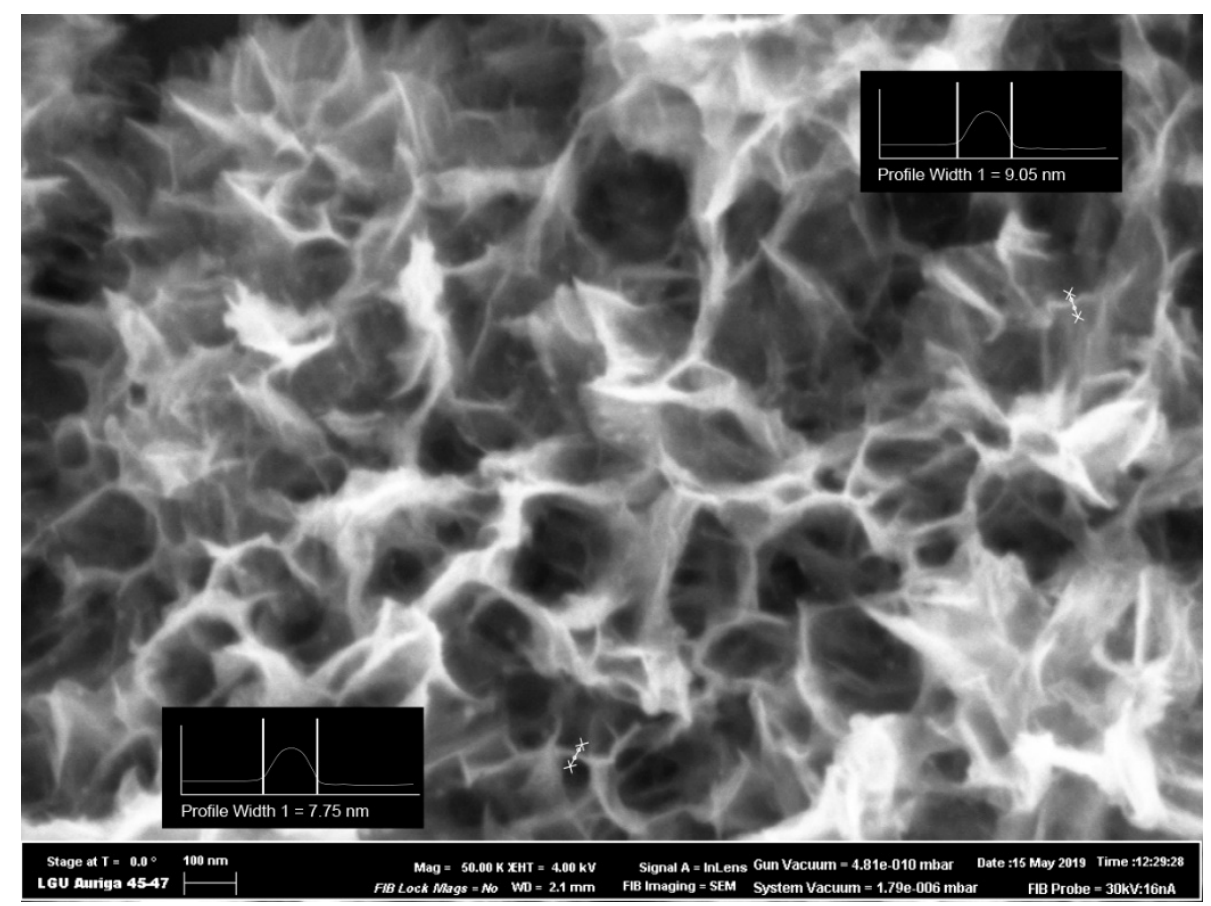

Figure S2. SEM images of interfacial $\gamma$-FeOOH film from solution side before Ag NPs synthesis.

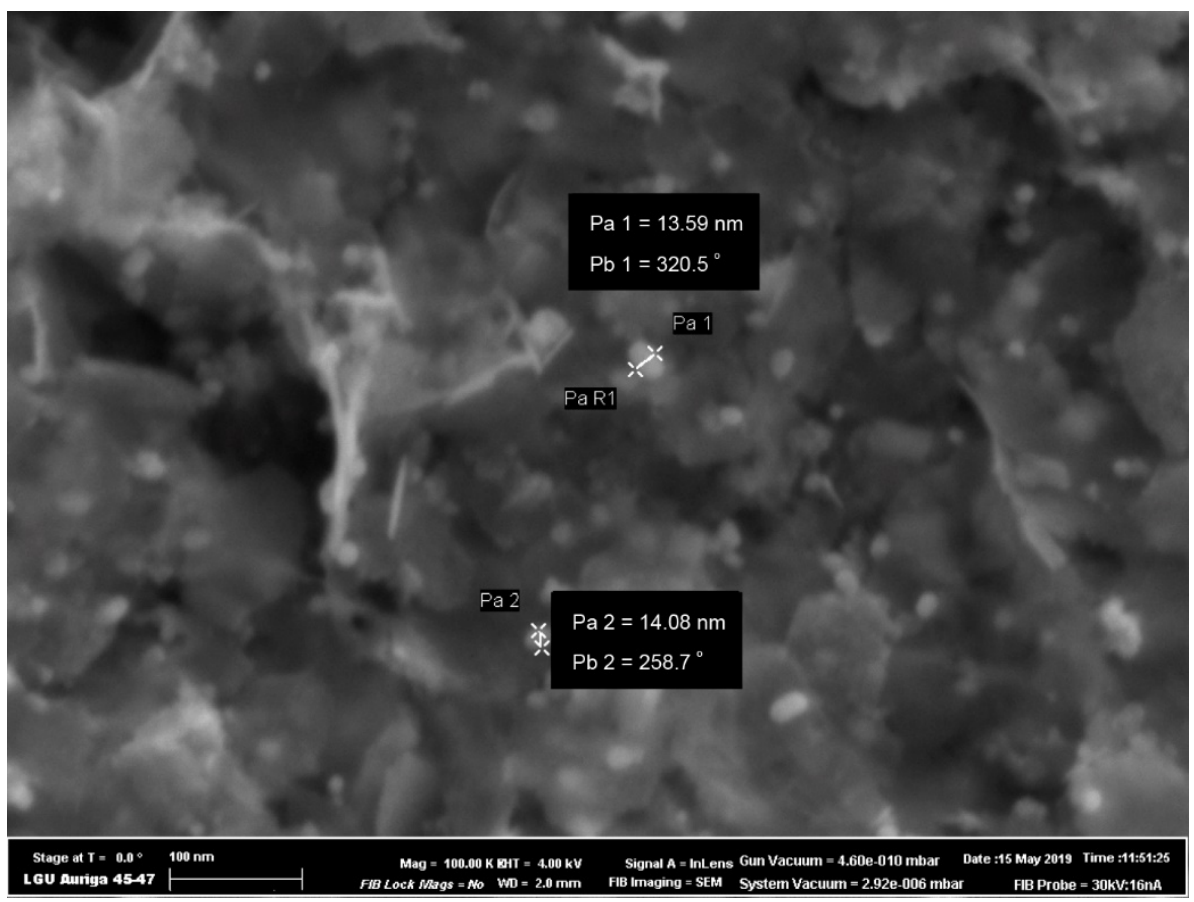

Figure S3. SEM images of interfacial $\gamma$-FeOOH film from solution side after one cycle of Ag NPs synthesis. 


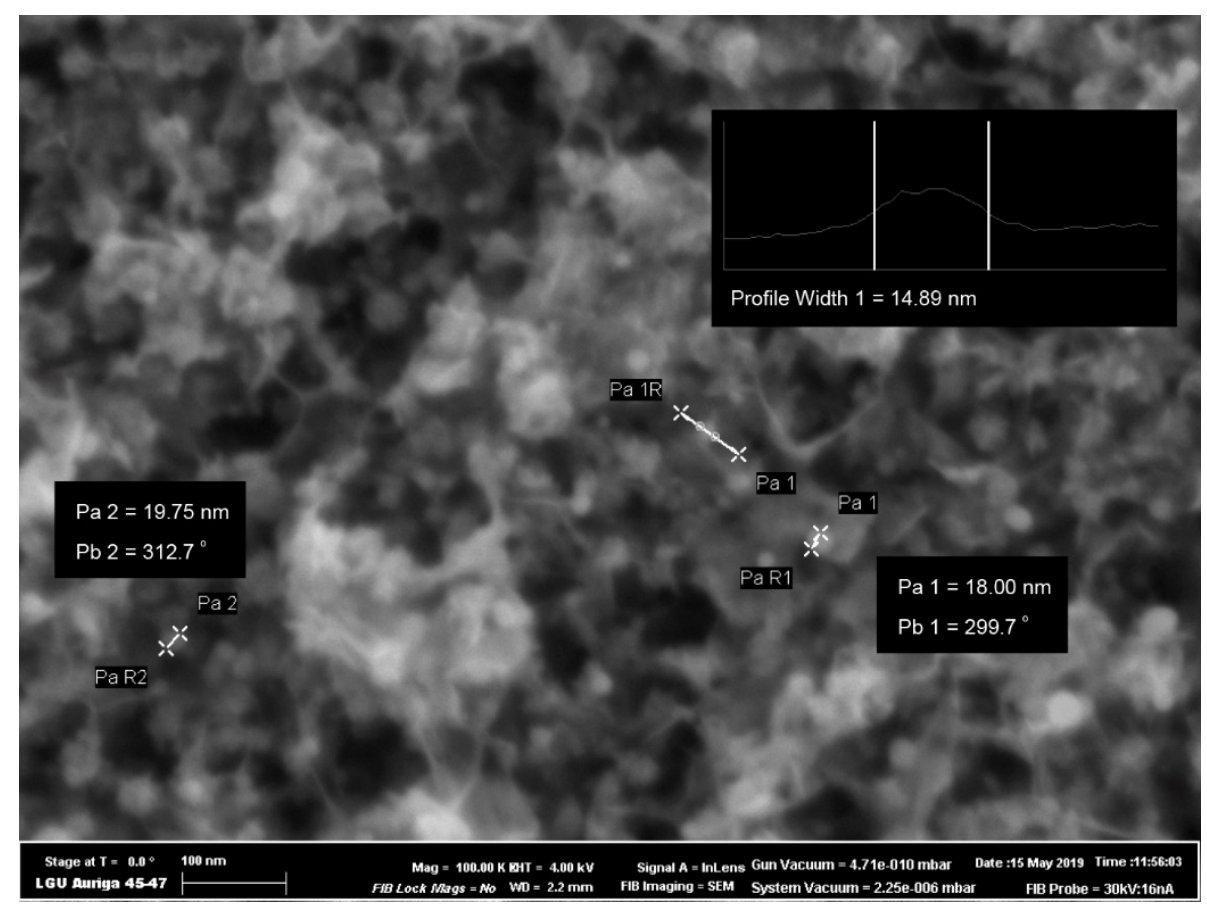

Figure S4. SEM images of interfacial $\gamma$-FeOOH film from solution side after two cycles of Ag NPs synthesis.

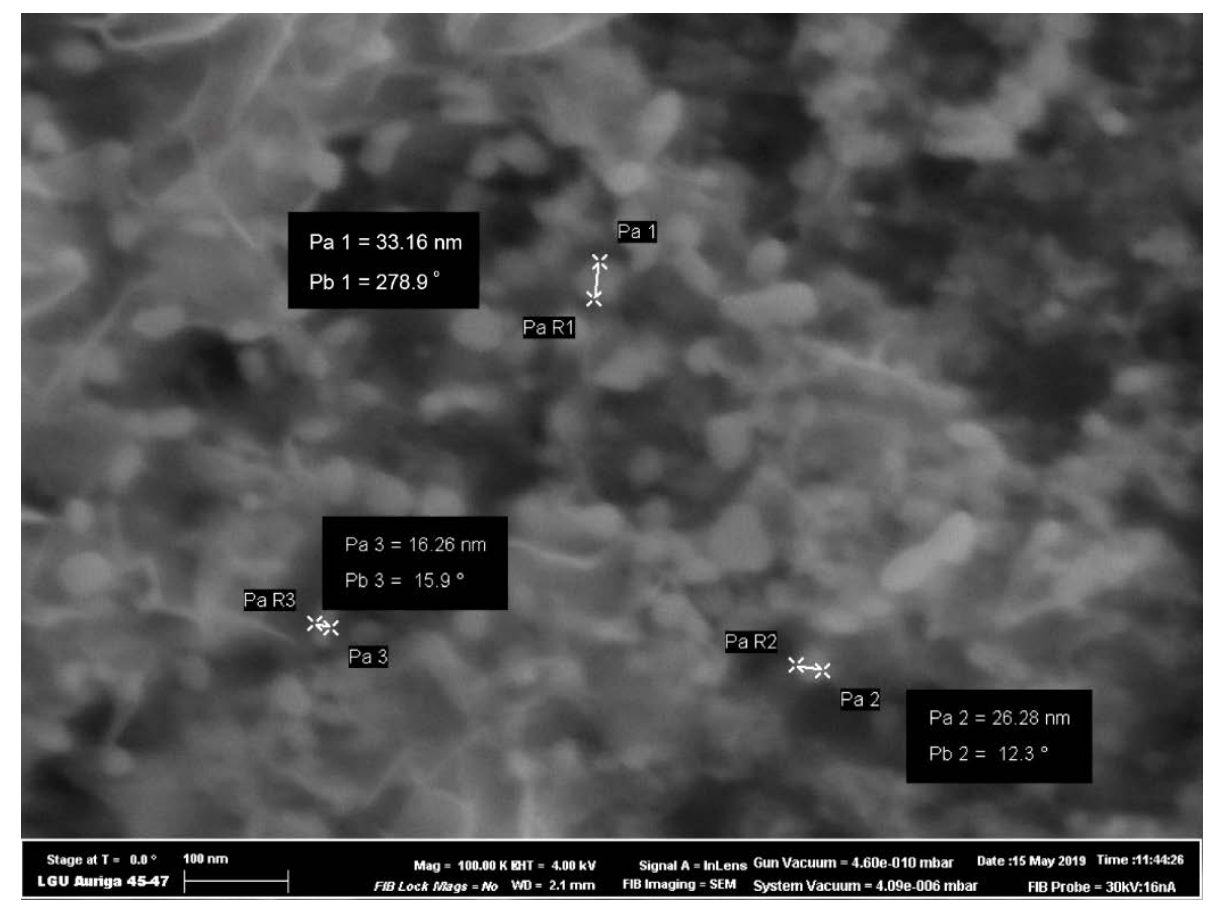

Figure S5. SEM images of interfacial $\gamma$-FeOOH film from solution side after 3 cycles of Ag NPs deposition. 